\title{
WAFER-LEVEL MECHANICAL AND ELECTRICAL INTEGRATION OF SMA WIRES TO SILICON MEMS USING ELECTROPLATING
}

Donato Clausi ${ }^{1}$, Henrik Gradin ${ }^{2}$, Stefan Braun ${ }^{2}$, Jan Peirs ${ }^{1}$, Dominiek Reynaerts ${ }^{1}$, Göran Stemme ${ }^{2}$ and Wouter van der Wijngaart ${ }^{2}$

${ }^{1}$ KUL - Katholieke Universiteit Leuven, Leuven, Belgium

${ }^{2} \mathrm{KTH}$ - Royal Institute of Technology, Stockholm, Sweden

\begin{abstract}
This paper reports on the wafer-level fixation and electrical connection of pre-strained SMA wires on silicon MEMS using electroplating, providing high bond strength and electrical connections in one processing step.

The integration method is based on standard micromachining techniques, and it potentially allows mass production of microactuators having high work density.

SEM observation showed an intimate interconnection between the SMA wire and the silicon substrate, and destructive testing performed with a shear tester showed a bond strength exceeding $1 \mathrm{~N}$.

The first Joule-heated SMA wire actuators on silicon were fabricated and their performance evaluated. Measurements on a $4.5 \times 1.8 \mathrm{~mm}^{2}$ footprint device show a $460 \mu \mathrm{m}$ stroke at low power consumption $(70 \mathrm{~mW})$.
\end{abstract}

\section{INTRODUCTION}

Shape memory alloy (SMA) actuators are known for the high force and work they generate. The force per unit surface can be in the hundreds of MPa, higher than for any other actuator type. In terms of work density SMAs outperform other active materials at the micro-scale, exceeding the work production per unit volume of other principles by at least an order of magnitude [1]. Furthermore, their surface to volume ratio increases with miniaturization, which results in enhanced cooling and higher bandwidth. Miniaturised actuators benefit also from a higher electrical resistance, thus requiring lower current when triggered by direct electrical heating.

Integration of SMAs to microsystems faces three main technical challenges: first, the SMA has to form a strong mechanical bond to the target structures to withstand the high forces and the high temperatures generated upon repeated actuation; second, mechanical and electrical connections should preferably be batch-manufactured using standard micromachining techniques, to achieve an overall cost reduction. Third, a bias mechanism is required to deform the SMA in martensitic state to achieve cyclic actuation. It is often difficult to implement this bias mechanism at the microscale [2].

The standard integration method of SMA materials to microsystems is based on sputter deposition of thin TiNi films on target MEMS structures, which is inherently a batchcompatible technique. Devices fabricated as such can be actuated by either direct or indirect heating [3]. However, difficult control of transformation temperatures and strains and limited mechanical robustness [4] reduce the range of application of structures driven by SMA films.

Commercially available bulk SMA materials feature strictly controlled transformation properties and allow a higher mechanical robustness of the microdevices. They display the highest energy efficiency when strained in pure tension, an order of magnitude larger as compared to torsion or bending loads [5]. However, they are typically integrated to microsystems by a pick-and-place approach, and electrical connections are difficult to realize in miniature devices.

Monolithic SMA actuators integrate both the actuating function and the reset mechanism in the same piece of material. Their material grain structure is changed by local annealing, either by direct Joule heating or by laser heating [6], to confer the desired functional properties to the targeted area: the annealed regions exhibit shape memory effect (SME), whereas the non-annealed parts display an elastic behavior and serve as bias spring. This approach allows avoiding assembly to a certain extent and it is not limited to out-of-plane bending actuators; however, it uses a vast amount of shape memory material while exploiting the SME only for small portions of it.

We reported on the wafer-level integration of SMA wires to silicon MEMS using adhesive bonding [7]. However, this approach required external heating for actuation, and the adhesive Si-to-SMA wire anchor was the point of failure due to the large stresses in the SMA during shape recovery. Another report [8] presented a per-piece fabrication method of catheters for medical applications based on connecting SMA coils to a stainless steel liner coil using electroplating. Placement of SMA wires at wafer-level was also performed with a conventional wire bonder [9].

In this work, we demonstrate a new wafer-level mechanical and electrical integration of SMA wires on arrays of silicon structures using standard electroplating. The fabrication sequence of SMA wires-on-silicon MEMS microactuators is discussed and both the SMA wire-to-silicon interface and the performance of the devices upon direct electrical heating are experimentally investigated.

\section{SMA WIRE ACTUATOR CONCEPT}

The microactuator's mechanical design (Figure 1) and the performance analysis for its two stable states were introduced previously [7]. The design consists of a fixed and a movable $\mathrm{Si}$ anchor, mechanically connected to i) two SMA wires that form a current loop, allowing direct joule heating, and ii) two Si cantilevers, serving as bias mechanism for the SMA wires. The wires are placed eccentrically onto the silicon cantilevers to allow out-of-plane actuation. The schematic operational states of the actuator are shown in Figure 2 . 


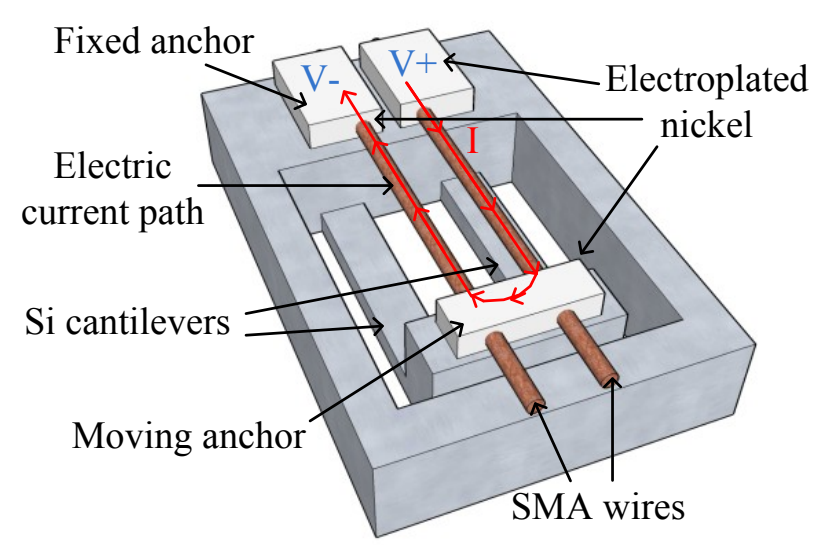

Figure 1: Illustration of the actuator. The SMA wires are mechanically connected to the two silicon anchors by electroplated nickel. The nickel features on the fixed anchor serve also as electrical contact pads, and together with the SMA wires and the nickel pad on the moving anchor form a current loop.

(a)

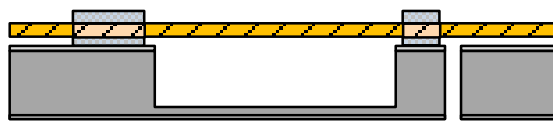

Cold state

(b)

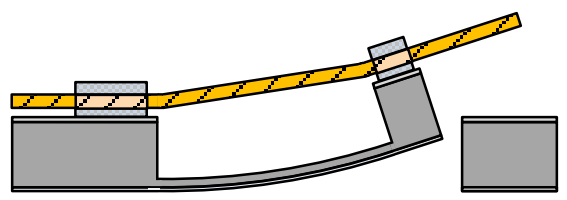

Hot state

Figure 2: Operational states of the actuator. At rest (no current flowing), the cantilevers stretch the SMA wires (a). Upon actuation, the SMA wires contract and bend the cantilevers, thus lifting the moving anchor $(b)$.

\section{FABRICATION}

Commercially available TiNi wires are strained using a dedicated frame [7] and then are oriented and integrated with silicon cantilevers that serve as bias springs. Integration of the pre-tensioned SMA wires to the micromachined structures is performed at wafer-level using electroplated nickel features, thus providing mechanical anchors between the SMA wires and the silicon, as well as direct electrical contacts to the wires. The fabrication of actuators is schematically illustrated in Figure 3. The size of the nickel fixtures is $1 \times 0.8 \mathrm{~mm}^{2}$ each on the fixed anchor and $0.4 \times 1.4 \mathrm{~mm}^{2}$ on the moving anchor, respectively. Test structures were also fabricated with the same process by integrating the SMA wires onto a plain silicon wafer.

A $7 \times 7$ array of silicon cantilever structures was fabricated starting with a thermally oxidized $320 \mu \mathrm{m}$ thick, 4 inch silicon wafer. The thermal oxide was patterned to be used as a hard mask. U-shaped cantilevers (as shown in Figure 1) were deep reactive ion etched and then covered with a $50 \mathrm{~nm}$ TiW adhesion layer and a $300 \mathrm{~nm}$ thick nickel layer by sputtering

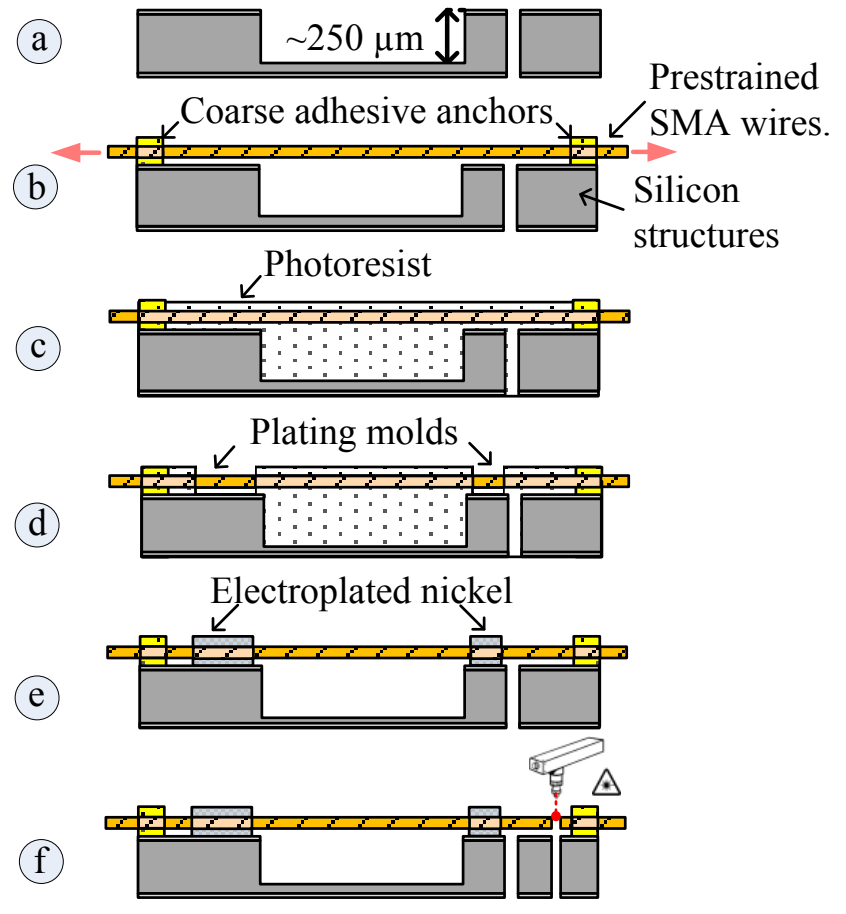

Figure 3: Schematic process flow. a) Front- and back-side DRIE to form silicon cantilevers. Sputter-deposit TiW and nickel. b) Transfer prestrained SMA wires onto the silicon wafer. c) Spin-on thick negative photoresist. d) Define electroplating molds. e) Nickel electroplating and stripping the resist. f) Dice chips and release the wires.

(Figure 3a).

SMA wires (Dynalloy Flexinol HT with a nominal diameter of $\phi 37.5 \mu \mathrm{m}$ ) were first deformed to $\sim 2.5 \%$ strain and then transferred onto the micromachined wafer using a dedicated metal frame [7]. Intermediate adhesive anchors, defined across the wafer using a coarse manual curing step, held the wires in place (Figure 3b). A thick layer of negative resist (NLOF 2070) was spin-coated onto the silicon wafer until it covered the SMA wires (Figure 3c). Electroplating molds were defined by photo-patterning and curing the resist layer (Figure 3d). Then the wafer was dipped in $\mathrm{HF}: \mathrm{H}_{2} \mathrm{O}$ (1:10) for $\sim 75 \mathrm{sec}$ to activate the exposed portions of the TiNi wires and of the sputtered nickel layer on the wafer surface. Thereafter the silicon-TiNi fixtures were formed on every actuator structure by nickel electroplating, and the resist was stripped (Figure 3e). The plating conditions were selected on the basis of guidelines provided by the manufacturer and of processing data available in literature [10]: $53^{\circ} \mathrm{C}$ temperature; $480 \mathrm{rpm}$ agitation; $4.2 \mathrm{~A} / \mathrm{dm}^{2}$ current density; $2 \mathrm{~h}$ plating time.

Due to the deep trenches in the micromachined wafer, imperfect resist coverage resulted on the actuators. As a result, excess nickel was deposited onto portions of the SMA wires. This problem was not encountered when fabricating the test structures. To remove the excess nickel from the SMA wires, the $\mathrm{Ni}$ anchors were protected by locally applied posi- 


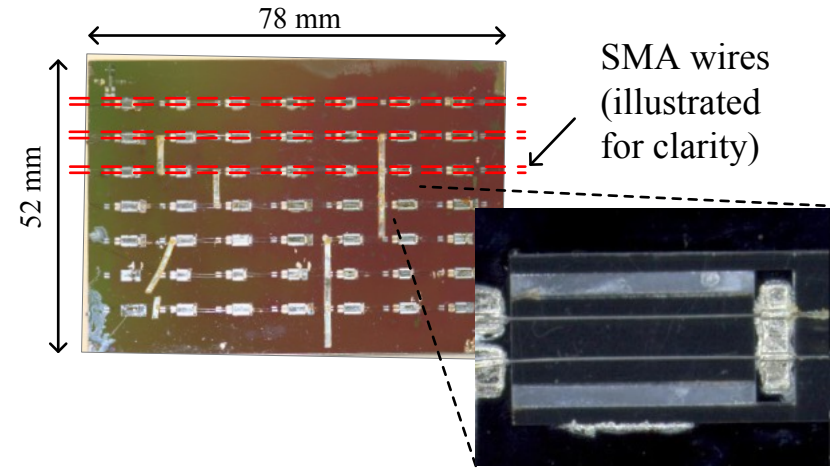

Figure 4: $7 x 7$ array of actuators. The 4 inch wafer was diced to accommodate it in 3 inch wire handling equipment.

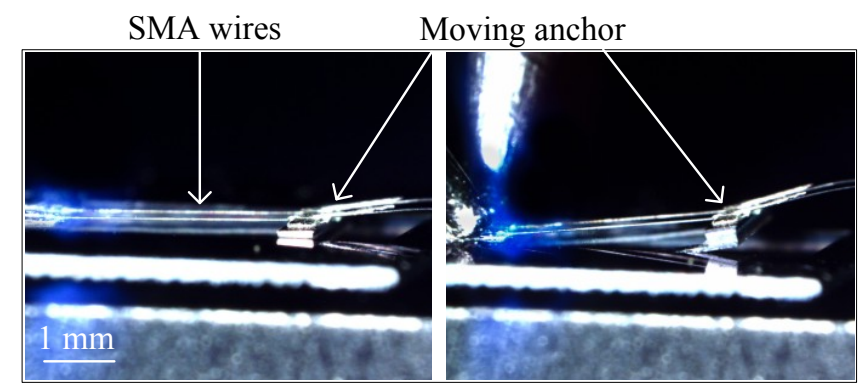

Figure 5: Sideview photographs of a device at rest (left) and during electric actuation (right).

tive resist, and the nickel deposits on the wires were removed by dipping the wafer in aluminum etchant. Thereafter the nickel seed layer and TiW adhesion layer were removed with aluminum etchant and $\mathrm{H}_{2} \mathrm{O}_{2}$ respectively, and then the wafer was diced into single chips (Figure $3 \mathrm{f}$ ).

The total yield was $\sim 50 \%$ for the array of microactuators and nearly $90 \%$ for the test structures. The main factors limiting the yield value for the actuators were the breaking of silicon structures during processing and the imperfect plating mask.

\section{EXPERIMENTAL RESULTS}

The actuators were successfully triggered by resistive (Joule) heating of the SMA wires. Repeatable actuation was observed, and photographs of a device at rest and during operation, respectively, are shown in Figure 5. The total footprint of this device was $4.5 \times 1.8 \mathrm{~mm}^{2}$.

The fixed anchors were contacted with a needle probe, and the current flowing through the SMA wires was recorded. An optical profiler employing white-light confocal interferometry (Veeco Wyco NT9300) was used to measure the actuator deflection. The tip displacement was inferred from colorcoded scans of the moving anchor, and the actuator deflection along a complete heating-cooling cycle was computed (Figure 6). The actuator featured deflections between $80 \mu \mathrm{m}$ and $540 \mu \mathrm{m}$, thus a net stroke of $460 \mu \mathrm{m}$, with a maximum

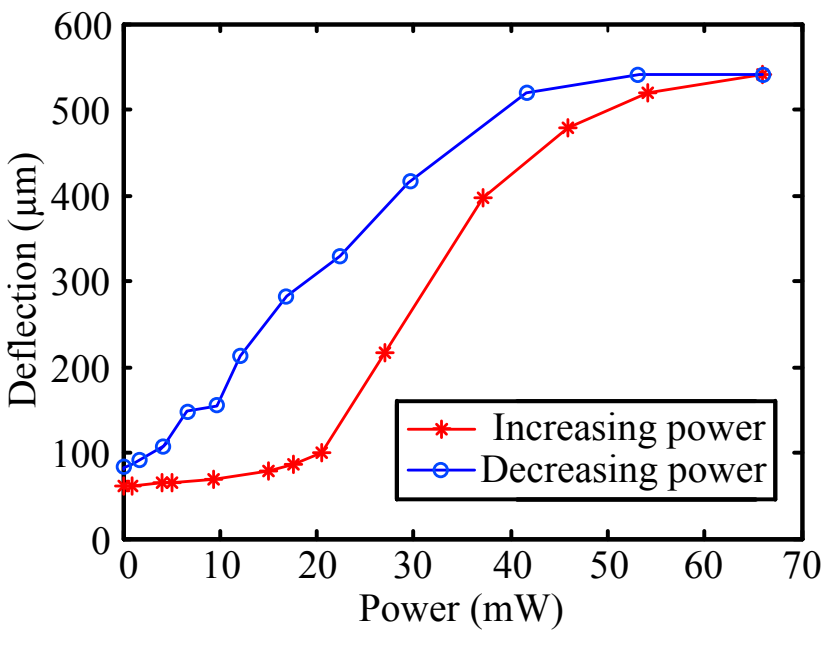

Figure 6: Optical profiler deflection measurements during electric actuation. Tip displacement values extracted from color coded optical profiler images.

estimated uncertainty below $10 \%$. The actuation current at full deflection was $82 \mathrm{~mA}$, and the corresponding voltage was $\sim 0.8 \mathrm{~V}$. Hence, the maximum input power was below $70 \mathrm{~mW}$. A hysteresis of about $10 \mathrm{~mW}$ is visible in Figure 6 between the two curves.

The bond between the SMA wires and the electroplated nickel on the test structures was investigated by scanning electron microscopy (SEM) and by mechanical testing using a tool for wire bonding testing (Dage PC 2400).

Prior to the SEM, a chip was embedded in polymer for mechanical stability, then a cross-section of the electroplated fixture of an SMA wire to a Si surface was prepared by mechanical polishing. A SEM picture of this cross section (Figure 7) shows homogeneous plating around the SMA wire, with intimate contact to the nickel and effective mechanical interconnection between the wire and the silicon anchor.

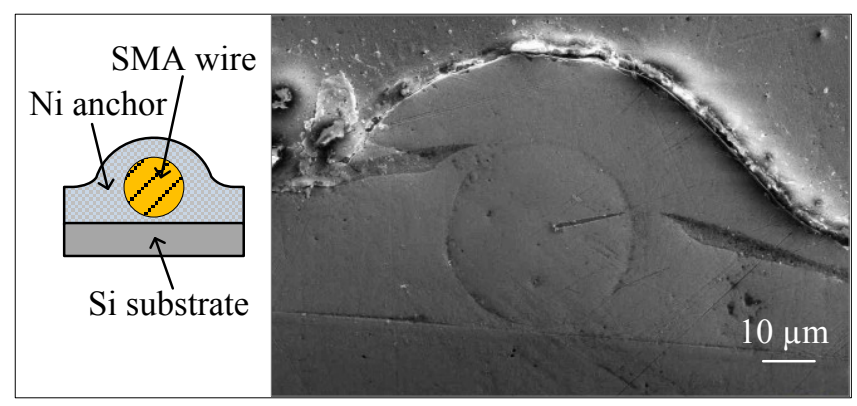

Figure 7: SEM picture of a polished cross-section of the electroplated fixture of an SMA wire to a Si surface. The structure was embedded in polymer for mechanical stability during preparation of the cross-section, and the cross-section was covered with a thin layer of gold. The wire has been homogeneously plated all around.

Destructive tests with the shear tester were performed on 
Electroplated nickel
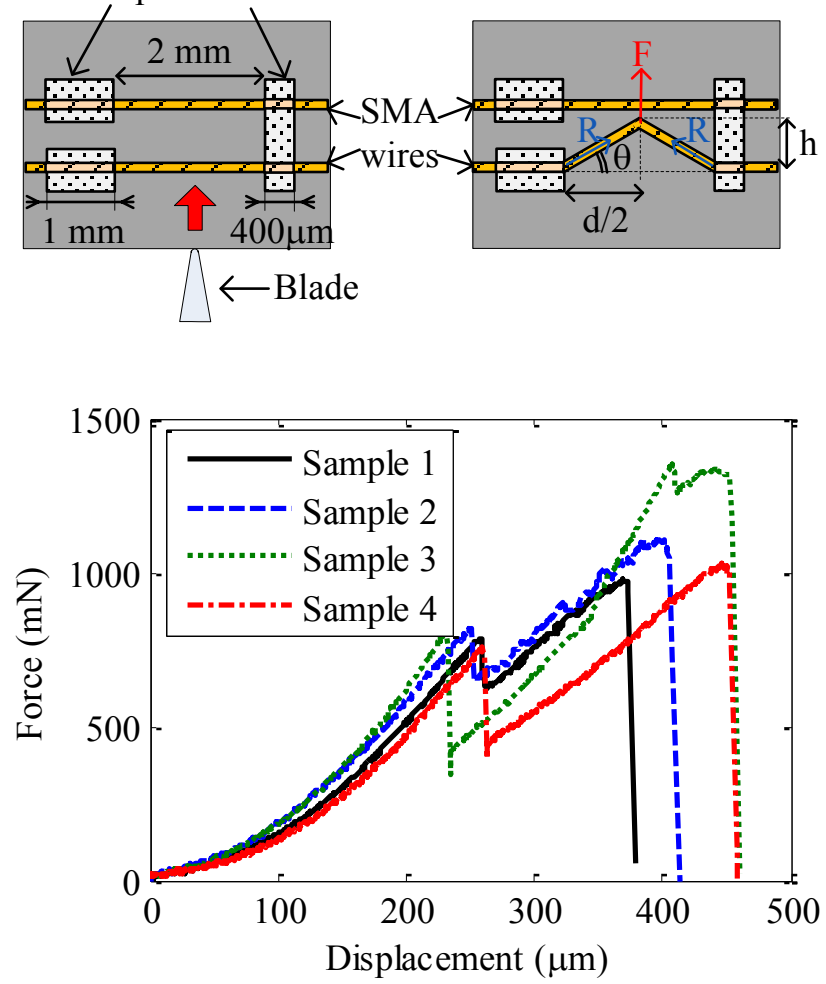

Figure 8: Bond strength measurements. The blade moves perpendicularly to the SMA wires, while the force is recorded.

the plain silicon test structures to avoid mechanical influence from, or failure of, silicon cantilevers. In this set-up a blade moved perpendicularly to the SMA wires at a constant speed, while the force was recorded. A schematic drawing of the test principle is shown in Figure 8, along with plots of blade displacement vs. force for different samples. In these tests, the SMA wire was sheared at forces above $1 \mathrm{~N}$, while the SMA-Ni-silicon bond remained intact.

\section{DISCUSSION AND CONCLUSIONS}

We successfully demonstrated the wafer-level integration of SMA wires to silicon microstructures using nickel electroplating, achieving both mechanical and electrical connection in the same process step.

An array of actuators was fabricated, and deflection measurements showed a tip displacement of $540 \mu \mathrm{m}$ at less than $70 \mathrm{~mW}$ power consumption.

Both SEM observation and destructive tests indicated a strong bond between SMA wire and underlying silicon, exceeding $1 \mathrm{~N}$ of perpendicularly applied force.

The integration process here developed relies on conventional micro machining techniques and it provides an efficient solution to some problems that have hindered the widespread diffusion of bulk SMA to MEMS, such as the lack of waferlevel integration methods and the difficult electrical contacting of the actuator material at small scale. High aspect ratio structures cause imperfect plating masks, which may result in a reduced yield or demand extra precautions. Future work will be devoted to the solution of these aspects.

The fabricated actuators showed high performance in terms of deflection and stroke, with displacements among the highest reported for actuators of comparable size [11].

\section{ACKNOWLEDGEMENTS}

The authors wish to thank J-KEM International AB for supplying the electroplating solution and for their help in the plating process.

\section{REFERENCES}

[1] J. van Humbeeck, D. Reynaerts, R. Stalmans: "Shape memory alloys: functional and smart", Proc. Actuator, Bremen, Germany, 1994, pp. 312-316

[2] Y. Bellouard: "Shape memory alloys for microsystems: A review from a material research perspective", Mater. Sci. Eng. A, vol. 481-482, pp. 582-589, 2008

[3] P. Krulevitch, A.P. Lee, P.B. Ramsey, J.C. Trevino, J. Hamilton, and M.A. Northrup: "Thin film shape memory alloy microactuators", J. Microelectromech. Syst., vol. 5, no. 4, pp. 270-282, Dec. 1996

[4] S. Miyazaki, M. Tomozawa, and H.Y. Kim: "Development of high-speed microactuators utilizing sputterdeposited TiNi-base shape memory alloy thin films", Proc. Actuators, Bremen, Germany, 2008, pp. 372-377

[5] D. Reynaerts, H. Van Brussel: "Design aspects of shape memory actuators", Mechatronics, vol. 8, Issue 6, pp. 635-656, Aug. 1998

[6] Y. Bellouard, T. Lehnert, J.E. Bidaux, T. Sidler, R. Clavel, an R. Gotthardt: "Local annealing of complex mechanical devices: A new approach for developing monolithic micro-devices", Mater. Sci. Eng. A, vol. 273-275, pp. 795-798, Dec. 1999

[7] D. Clausi, H. Gradin, S. Braun, J. Peirs, G. Stemme, D. Reynaerts, W. van der Wijngaart: "Design and WaferLevel Fabrication of SMA Wire Microactuators on Silicon", J. of Microelectromech. Syst., Vol. 19, No. 4, Aug. 2010, pp 982-991

[8] Y. Haga, M. Esashi, S. Maeda: "Bending, torsional and extending active catheter assembled using electroplating", Proc. IEEE MEMS 2000, pp. 181-185

[9] A.C. Fischer, H. Gradin, S. Braun, S. Schroeder, G. Stemme, and F. Niklaus: "Wafer-level integration of NiTi shape memory alloy wires for the fabrication of microactuators using standard wire bonding technology", Proc. IEEE MEMS 2011

[10] M.J. Madou: "Fundamentals of MICROFABRICATION, The Science of Miniaturization", Second edition, (2002) pp. 350-352

[11] D.J. Bell, T.J. Lu, N.A. Fleck, and S.M. Spearing: "MEMS actuators and sensors: observations on their performance and selection for purpose", J. Micromech. Microeng. 15 (2005), S153-S164 